\title{
VARIABILIDADE PLUVIOMÉTRICA NO MUNICÍPIO DE ACOPIARA/CE
}

\author{
Cicera Celiane Januário da Silva ${ }^{(a)}$, Joyce Ferreira Gomes ${ }^{(b)}$, Juliana Maria Oliveira Silva ${ }^{(c)}$ \\ (a) Graduanda em Geografia e bolsista de Iniciação Científica - PIBIC/URCA, celianejanuario@gmail.com, \\ (b) Estagiária no Laboratório de Análise Geoambiental - LAGEO, URCA, joyceferreira234@ yahoo.com, \\ (c) Orientadora, Professora do Departamento de Geociências/URCA, juliana.oliveira@ urca.br
}

Eixo: 3. Climatologia em diferentes níveis escalares: mudanças e variabilidades.

\begin{abstract}
Resumo
Esta pesquisa tem como objetivo identificar os anos chuvosos, secos e normais da série histórica do Município de Acopiara localizado no centro sul do Estado do Ceará, no período entre 1974 a 2015, comparando com as condições oceânicas do Pacifico (El Niño e La Niña) e Atlântico (dipolo) que possam ter influenciado no padrão pluviométrico da área de estudo. Para a classificação dos anos executou-se o método Box Plot. Dos anos analisados, 19 encontram-se na categoria normal, 11 no padrão seco e 11 no chuvoso e que os fenômenos oceânicos tiveram influência na distribuição das chuvas na série histórica. Conclui-se que é de grande importância estudar essa variabilidade para compreender como a interação do oceano e a atmosfera inibem ou favorecem a ocorrência das chuvas nos municípios do semiárido nordestino.
\end{abstract}

Palavras-chave: Variabilidade pluviométrica, oceano, semiárido.

\section{Introdução}

O município de Acopiara (“coordenadas $6^{\circ} 05^{\prime} 43^{\prime}$ " Lat S e $39^{\circ} 27^{\prime} 09^{\prime}$ " Long O) localiza-se no centro sul do Estado do Ceará, distante $353 \mathrm{Km}$ da capital Fortaleza. Apresenta um Clima Tropical Quente Semiárido. A altitude encontra-se em torno de 317 metros com as formas de relevo predominante da Depressão Sertaneja e Maciço Residual, os principais tipos de solos são os Neossolos Aluviais, Litólicos e Argissolos e vegetação do tipo Caatinga Arbustiva (IPECE, 2016). 

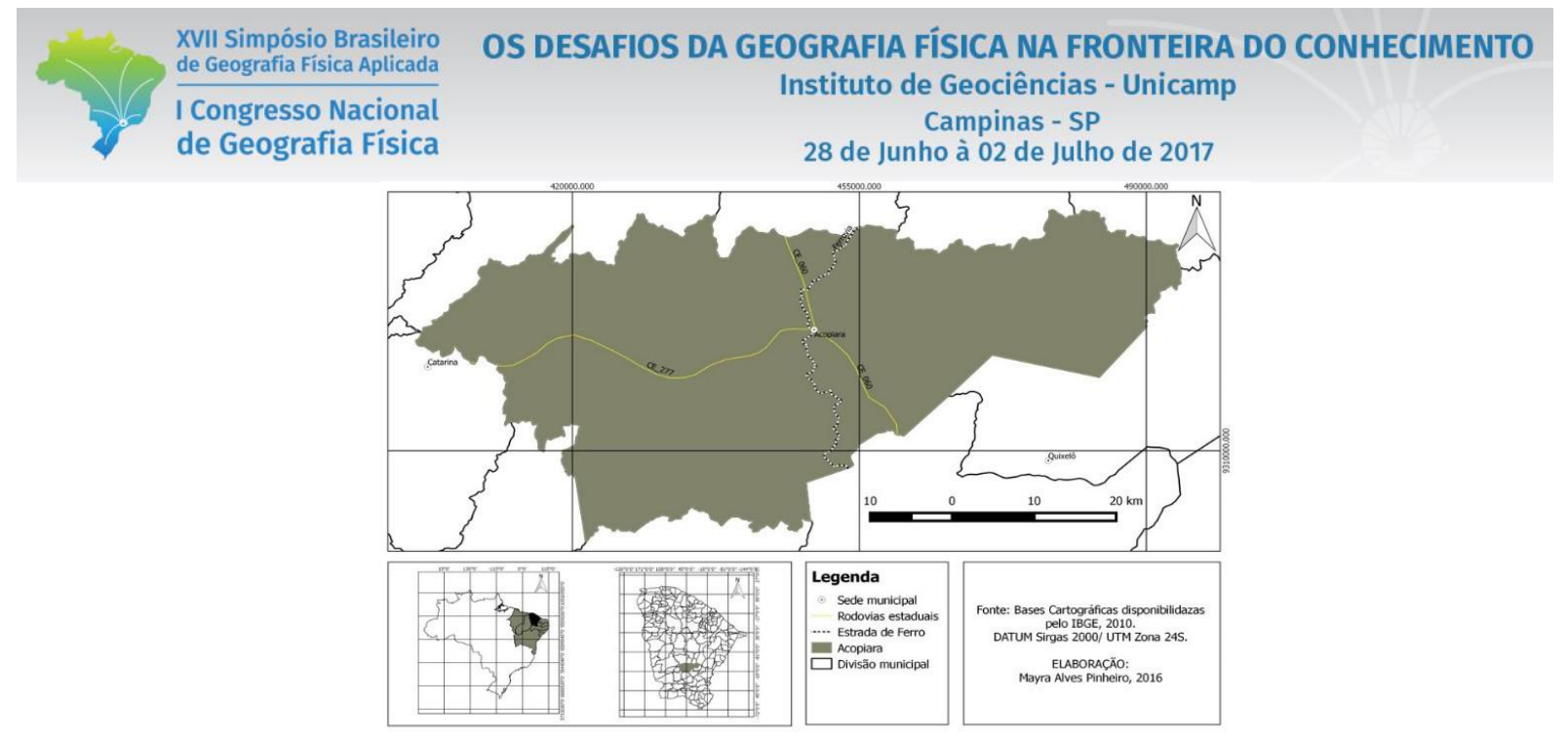

Figura 1 - Localização da área de estudo. Fonte: PINHEIRO, M. A.

O objetivo desta pesquisa consiste em identificar os anos chuvosos, secos e normais da série histórica de chuva do município (período entre 1974 e 2015) e comparar com as condições oceânicas do Pacífico e Atlântico que possam ter influenciado no padrão pluviométrico.

O El Niño caracteriza-se pelo aquecimento anormal do Oceano Pacífico causando uma alteração da Célula de Walker e este aquecimento se torna acima da média histórica, desenvolvendo uma anomalia positiva nos valores de temperatura da superfície do mar (NÓBREGA e SANTIAGO, 2014). Em anos de ocorrência desse fenômeno e dependendo de sua intensidade, os valores de precipitação observados no semiárido nordestino podem apresentar-se abaixo da média, favorecendo então um ano seco ou muito seco, principalmente quando acontece com o dipolo positivo do Atlântico (FERREIRA e MELO, 2005).

As anomalias da Temperatura da Superfície do mar no Oceano Atlântico podem gerar o padrão dipolo positivo (desfavorável às chuvas no Nordeste) ou negativo (favorável às chuvas). De acordo com Ferreira e Melo (2005) O fenômeno La Niña quando associado ao dipolo negativo do Atlântico (favorável às chuvas), é normalmente responsável por anos considerados normais, chuvosos ou muito chuvosos na região.

\section{Metodologia}

A metodologia constou em consultas bibliográficas realizadas sobre o tema, tabulação dos dados, construção de gráficos e interpretação dos dados. Os valores de precipitação utilizados foram obtidos através da homepage da Fundação Cearense de Meteorologia e Recursos Hídricos (FUCEME). Os anos de intensidade do fenômeno El Niño e da La Niña, Dipolo do Atlântico foram obtidos no site do CPTEC/INPE, FUNCEME e da bibliografia disponível em artigos como de Nóbrega e Santiago (2014). Para a classificação dos anos em normais, chuvosos e secos executou-se o método Box Plot que segundo Galvani e Luchiari (2005) é uma técnica estatística que permite identificar para os totais mensais de 


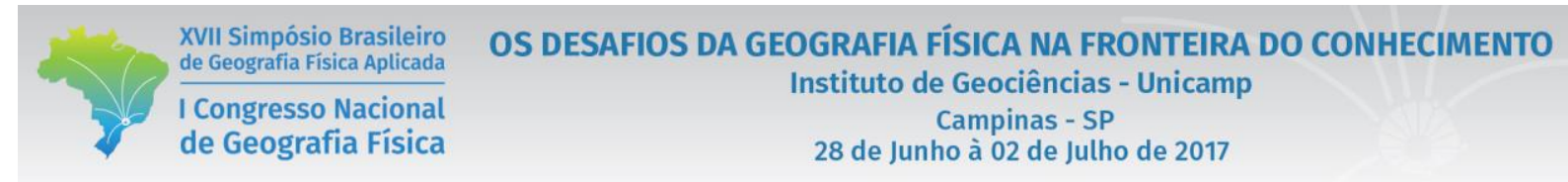

precipitação os valores máximos, os mínimos, a mediana, o primeiro quartil e o terceiro quartil. O gráfico do Box Plot foi realizado no software Action Pro e considerou-se que os anos secos estavam localizados entre o valor mínimo e o primeiro quartil $(366 \mathrm{~mm}-607 \mathrm{~mm})$, o ano normal entre o $1^{\circ}$ quartil, mediana e o $3^{\circ}$ quartil $(607 \mathrm{~mm}-904 \mathrm{~mm})$ e o ano chuvoso a partir do valor máximo em diante (>904mm).

\section{Resultados}

O gráfico 01 demonstra a distribuição da precipitação anual da série histórica entre 1974-2015. A média do município representada pela linha laranja apresenta $756 \mathrm{~mm}$. A variação da precipitação em relação à média apresenta alguns anos com valores acima desta, tendo como destaque o ano de 1985 com 1469mm, ou abaixo da média, como é o caso de 1993 (366mm). As chuvas no município têm início em janeiro, mas são nos meses de fevereiro a maio que apresenta os maiores valores, coincidindo com a atuação da Zona de Convergência Intertropical (ZCIT), principal sistema meteorológico causador de chuva no Ceará e consequentemente no município. A partir do mês de maio a precipitação começa a diminuir, chegando em valores muito baixos de junho a dezembro. O mês de maior precipitação é o de março com 168,47mm.

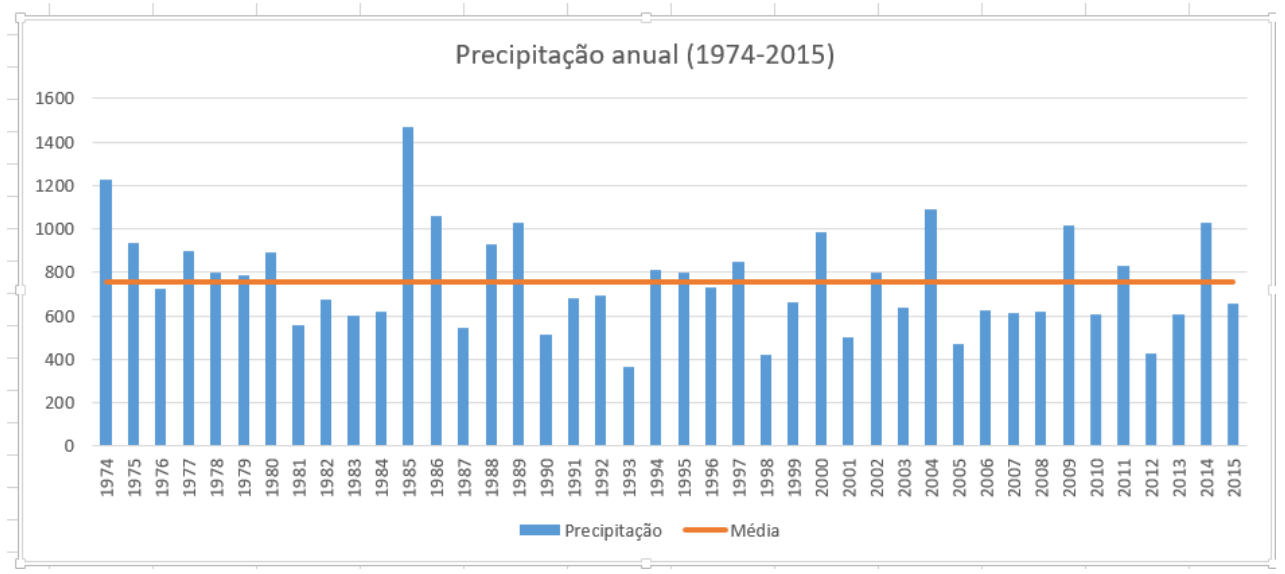

Figura 2 - Precipitação Pluviométrica total anual de 1974 à 2015.

Fonte: Funceme, 2016.

A tabela 01 demonstra a classificação para cada ano estudado, o total pluviométrico e a ocorrência de eventos de El Niño, La Niña e Dipolo do Atlântico para o município de Acopiara.

Tabela I - Classificação do padrão pluviométrico, Intensidade El Niño/La Niña e Dipolo do Atlântico para a área de estudo

\begin{tabular}{|c|c|c|c|c|c|}
\hline ANOS & TOTAL & CLASSIFICAÇÃO & El niño & La Ninã & Dipolo \\
\hline 1974 & 1226 & Chuvoso & Neutro & Forte & Negativo \\
\hline 1975 & 936 & Chuvoso & Fraco & Forte & Neutro \\
\hline 1976 & 722,2 & Normal & Fraco & Forte & Neutro \\
\hline 1977 & 896 & Normal & Fraco & Neutra & Negativo \\
\hline
\end{tabular}




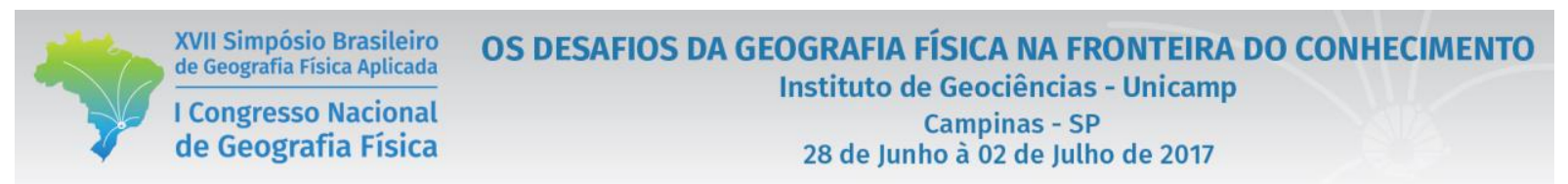

\begin{tabular}{|c|c|c|c|c|c|}
\hline 1978 & 799 & Normal & Fraco & Neutra & Positivo \\
\hline 1979 & 785 & Normal & Fraco & Neutra & Positivo \\
\hline 1980 & 890 & Normal & Fraco & Neutra & Positivo \\
\hline 1981 & 557,5 & Seco & Neutro & Neutra & Positivo \\
\hline 1982 & 676,5 & Normal & Forte & Neutra & Neutro \\
\hline 1983 & 601,4 & Seco & Forte & Fraca & Positivo \\
\hline 1984 & 616,4 & Seco & Neutro & Fraca & Negativo \\
\hline 1985 & 1469 & Muito chuvoso & Neutro & Fraca & Negativo \\
\hline 1986 & 1058 & Chuvoso & Moderado & Neutra & Negativo \\
\hline 1987 & 545 & Seco & Moderado & Neutra & Neutro \\
\hline 1988 & 928,5 & Chuvoso & Moderado & Forte & Negativo \\
\hline 1989 & 1025,7 & Chuvoso & Neutro & Forte & Negativo \\
\hline 1990 & 510,5 & Seco & Forte & Neutra & Neutro \\
\hline 1991 & 684,1 & Normal & Forte & Neutra & Negativo \\
\hline 1992 & 692,5 & Normal & Forte & Neutra & Positivo \\
\hline 1993 & 366 & Seco & Forte & Neutra & Neutro \\
\hline 1994 & 809 & Normal & Moderado & Neutra & Negativo \\
\hline 1995 & 799,9 & Normal & Moderado & Fraca & Negativo \\
\hline 1996 & 731,5 & Normal & Neutro & Fraca & Negativo \\
\hline 1997 & 846,6 & Normal & Forte & Neutra & Positivo \\
\hline 1998 & 421 & Seco & Forte & Neutra & Neutro \\
\hline 1999 & 664,2 & Normal & Neutro & Moderada & Negativo \\
\hline 2000 & 984,2 & Chuvoso & Neutro & Moderada & Negativo \\
\hline 2001 & 504 & Seco & Neutro & Neutra & Neutro \\
\hline 2002 & 797 & Normal & Moderado & Neutra & Negativo \\
\hline 2003 & 640 & Normal & Moderado & Neutra & Negativo \\
\hline 2004 & 1093 & Chuvoso & Fraco & Neutra & Neutro \\
\hline 2005 & 468,6 & Seco & Fraco & Neutra & Positivo \\
\hline 2006 & 626 & Normal & Fraco & Neutra & Neutro \\
\hline 2007 & 612,3 & Normal & Fraco & Forte & Positivo \\
\hline 2008 & 618,4 & Normal & Neutro & Forte & Negativo \\
\hline 2009 & 1013,2 & Chuvoso & Fraco & Neutra & Negativo \\
\hline 2010 & 606,2 & Seco & Fraco & Neutra & Neutro \\
\hline 2011 & 828,9 & Chuvoso & Neutro & Fraca & Negativo \\
\hline 2012 & 427,3 & Seco & Neutro & Fraca & Positivo \\
\hline 2013 & 608 & Chuvoso & Neutro & Neutro & Positivo \\
\hline 2014 & 1028,5 & Chuvoso & Neutro & Neutro & Positivo \\
\hline 2015 & 657 & Normal & Moderado & Neutro & Positivo \\
\hline
\end{tabular}

Fonte: Organização própria com base em Nóbrega e Santiago, 2014 / CPTEC/INPE/Funceme

O ano normal ou habitual é compreendido quando o total pluvial situa-se dentro dos desvios médios padrão (SIMIONI et al, 2008). Desta forma, foram contabilizados com o Box-Plot dezenove anos (1976, 1977, 1978, 1979, 1980, 1982, 1991, 1992, 1994, 1995, 1996, 1997, 1999, 2002, 2003, 2006, 2007, 2008 e 2015). Desses anos escolheu-se o ano de 2008 para identificar a atuação dos fenômenos oceânicos. Neste ano a pluviosidade atingiu 618,4mm. A La Niña encontrava-se Forte e o Dipolo Negativo, favorecendo as chuvas no município. Apesar de que a atuação conjunta favorável dos dois poderia ter influenciado para um padrão mais chuvoso, na área de estudo a distribuição das chuvas ficou dentro da normalidade. 
Na classificação dos anos chuvosos identificaram-se onze anos $(1974,1975,1985,1986,1988,1989$, 2000, 2004, 2009, 2011, 2013 e 2014), tendo destaque o ano de 1985, onde a precipitação alcançou $1469 \mathrm{~mm}$. Neste ano o El Niño estava neutro e La Niña fraca e o Dipolo Negativo. Essa fase Negativa do Atlântico favoreceu as chuvas em todo o Estado do Ceará, considerado um dos anos mais chuvosos para o estado e consequentemente para o município estudado.

Os anos secos encontrados no município com precipitação abaixo da média, contabilizou onze anos (1981, 1983, 1984, 1987, 1990, 1993, 2001, 2005, 2010 e 2012), sendo escolhido para análise o ano 1993, sendo considerado um ano de menor pluviosidade com apenas 366mm. Neste ano o El Niño encontrava-se Forte e o Dipolo Neutro, desfavorecendo as chuvas na região, caracterizando como o maior ano da série histórica com menor pluviosidade.

\section{Conclusão}

Com a realização deste trabalho demonstrou-se que há uma relação dos fenômenos oceânicos com o padrão de chuvas no município. A partir das observações feitas percebemos que quando ocorre o fenômeno El Niño favorece chuvas abaixo da média, mas observou-se também, que alguns anos da série mesmo com a atuação do El Niño, o padrão pluviométrico manteve-se Normal. Já em anos de La Niña ou Dipolo Negativo geralmente as chuvas ficam entre a média ou acima da média. Nesse sentido é importante estudar e ampliar as análises para compreender a distribuição irregular das precipitações ao longo da série e como esses oceanos podem exercer influência na variabilidade pluviométrica da área.

\section{Bibliografia}

CPTEC. Centro de Previsão de Tempo e Estudos Climáticos. El Niño e La Niña. Disponível em http://enos.cptec.inpe.br/. Acessado em 01 de novembro de 2016.

Disponível em http://www.ipece.ce.gov.br/. Acessado em 03 de novembro de 2016.

FERREIRA, A. G. F.; MELLO, N. G. da S. Principais sistemas atmosféricos atuantes sobre a Região Nordeste do Brasil e a influência dos oceanos pacífico e Atlântico no clima da região. Revista Brasileira de Climatologia, vol.1, $\mathrm{n}^{\circ} 1,2005$.

FUNCEME, Fundação Cearense de Meteorologia e Recursos Hídricos. Base de dados pluviométricos. Fortaleza: FUNCEME. Disponível em 〈www.funceme.br >. Acesso em 08 de agosto de 2016.

IPECE. Instituto de Pesquisa e Estratégia Econômica do Ceará. Perfil Básico Municipal de Acopiara.

NÓBREGA, R.S; SANTIAGO, G. A. C. F. Tendência de temperatura na superfície do mar nos oceanos Atlântico e Pacífico e a variabilidade de precipitação em Pernambuco. Revista Mercator (Fortaleza. Online), v. 13, p. 107-118, 2014.

SIMIONI, JOÃO PAULO ET AL. Caracterização da precipitação pluviométrica na bacia hidrográfica do rio Ibicuí, RS. Acessado 15 de Janeiro de 2017. Revista do Departamento de Geografia - USP, Vol. 28, p. 112-133. 2014. 